\title{
Utber die Untersuchung von Acetylverbindungen und eine neue Methode zur Analyse der Fette.
}

\author{
Von R. Benedikt und F. Ulzer.
}

(Aus dem Laboratorium für allgemeine und analytische Chemie an der k. k. technischen Hochschule in Wien.)

(Vorgelegt in der Sitzung am 13. Jänner 1887.)

In den meisten Acetylverbindungen, welche sich von Körpern der Fettreihe ableiten, lässt sich die Acetylbestimmung sehr leicht in der Weise vornehmen, dass man durch Titration die Alkalimenge bestimmt, welche zur vollständigen Verseifung benöthigt wird. Weniger allgemein anwendbar ist diese Methode bei aromatischen Verbindungen, indem sie nämlich voranssetzt, dass sich die Muttersubstanz der Acetylverbindung beim Kochen mit alkoholischem Kali entweder gar nicht oder in so glatter Weise zersetze, dass die dazu nöthige Kalimenge genau berechnet oder durch den Versuch ermittelt werden kann.

Zur Ausführung des Verfahrens bedient man sich mit Vortheil der von Köttstofer ${ }^{1}$ zur Bestimmung der Verseifungszahl von Fetten, insbesondere von Butter und Buttersurrogaten gegebenen Vorschrift, welche, von verschiedenen Autoren modificirt, gegenwärtig in folgender Weise ausgeführt wird:

Man bereitet folgende Lösungen:

1. Titrirte Salzsäure, am besten circa $1 / 2$-normal. Der Titer muss mit grosser Sorgfalt gestellt sein.

2. Titrirte Kali- oder Natronlauge, ebenfalls circa $1 / 2$-normal.

3. Alkoholische Kalilauge. Man löst ungefähr $30 \mathrm{Grm}$. aus Alkohol gereinigten Kalilydrates in möglichst wenig Wasser und verdunnt mit fuselfreiem Weingeist auf 1 Liter. Man lässt die Lösung zwei Tage stehen und filtrirt sie in eine Flasche, in deren

1 Zeitschrift f. analyt. Chemie 18, 199. 
Hals man einen Kautschukpfropfen eingepasst hat, durch dessen einfache Bohrung eine bis an den Boden der Flasche reichende $25 \mathrm{CCm}$.Pipette hindurchgeht. Dieselbe ist an ihrem oberen Ende mit einem Stückchen Schlauch und Quetschhahn verschlossen. Die Flasche wird an einem gleichmässig warmen Orte aufbewahrt. War der Weingeist rein, so nimmt die Flüssigkeit auch nach längerem Stehen keine braune, sondern nur eine gelbliche Färbung an.

4. Alkoholisehe Phenolphtalëinlösung mit cirea 1 Grm. des Farbstoffes in $200 \mathrm{CCm}$. Weingeist. In den Kautschukpfropfen der Flasche ist eine $1 \mathrm{CCm}$.-Pipette eingesetzt.

Die Menge der zur Analyse zu verwendenden Substanz hängt von ihrem Acetylgehalt ab, sie soll so gewählt werden, dass zu ihrer Verseifung $12-20 \mathrm{CCm}$. der alkoholischen Kalilange verbraucht werden. Von Substanzen mit grosem Acetylgehalt kann man zur Erböhıng der Genauigkeit des Resultates die doppelte Menge nehmen, nur müssen dann $50 \mathrm{CCm}$. der alkoholischen Kalilauge an Stelle der unten vorgeschriebenen $25 \mathrm{CCm}$. zur Verseifung verwendet werden.

Die Substanz (meist 1-2 Grm.) wird in einem weithalsigen Kölbchen von $100-150 \mathrm{CCm}$. Inhalt abgewogen. Danu füllt man die in die Vorrathsflasche eingesetzte $25 \mathrm{CCm}$.-Pipette genan bis zur Marke mit alkoholischer Kalilauge und lässt dieselbe in den Kolben fliessen. Ist die Hauptmenge abgelanfen, so lässt man noch einige Zeit abtropfen, wobei man dienachfliessenden Tropfen uählt, um bei allen folgenden Versuchen die Pipette genau bis zu demselben Punkte entleeren zu können. Sodann erhitzt man den mit einem kleinen Trichter bedeckten Kolben 15 Minuten auf dem Wasserbade zum schwachen Sieden, fiugt $1 \mathrm{CCm}$. Phenolphtalëinlösung zu und titrirt mit Salzsäure bis zur Eutfärbung. zurück.

In genau derselben Weise stellt man den Titer der alkoholisehen Kalilösung. Man erwärmtalso wieder $25 \mathrm{CCm}$. derselben 15 Minuten auf dem Wasserbade und titrirt sodann mit Salzsäure. Da ihr Titer etwas veränderlich ist, so wird er vor jeder Versuchsreihe neu gestellt.

Aus der Differenz zwischen der zweiten und ersten Titrirung lässt sich die Kalimenge leicht berechnen, welche zur Verseifung 
der Substanz nothwendig war, und daraus ergibt sich weiter die Verseifungszahl, d. i. die Anzahl Milligramme Kalihydrat, welche zur Verseifung von $1 \mathrm{Grm}$. der acetylirten Substanz yerbrancht werden.

Ist die zu untersuchende Verbindung eine Säure, so wird ein Theil des Kalihydrates zur Absättigung der Carboxylgruppe, der Rest zur eigentlichen Verseifung, d. i. zur Neutralisation der entstehenden Essigsäure verwendet. In solchen Fällen kann die Verseifungszahl als die Snmme der "Säurezahl" nnd der "A cetyl\%ahl" angesehen werden.

\section{Moleculargewichtsbestimmung von Fettalkoholen. ${ }^{1}$}

Das Molekulargewicht höherer Fettalkohole, ferner des Cholesterins und ähnlicber Verbindungen lässt sich durch Ermittlung der Verseifungszahl ihrer Essigsäureester ebenso sicher und auf einfachere Weise bestimmen, als nach der schönen Methode von C. Hell. ${ }^{2}$

Man stellt durch Kochen mit dem gleichen bis doppelten Gewichte Essigsä̈reanhydrid den Essigester her, giesst die heisse Mischung in Wasser ein und kocht wiederholt mit Wasser aus. Das Product kann endlich noch aus Weingeist umkrystallisirt werden. Sodann bestimmt man die Verseifungszahl.

Als Beispiel sei die Moleculargewichtsbestimmung des Cetylalkohols angeführt:

Titer der Salzsäure: $1 \mathrm{CCm} . \mathrm{HCl}=0.02577 \mathrm{Grm} . \mathrm{KHO}$.

$25 \mathrm{CC}$. alkoholische Kalilauge $=30 \cdot 35 \mathrm{CCm}$. HCl.

$2 \cdot 5715 \mathrm{Grm}$. Cetylessigsäureester wurden mit $25 \mathrm{CCm}$. alkoholischer Kalilauge verseilt, zum Zuritcktitriren $10 \cdot 90 \mathrm{CCm}$. $\mathrm{HCl}$. verbraneht.

Somit ist die $30 \cdot 35-10 \cdot 90=19 \cdot 45 \mathrm{CCm}$. Salzsäure entsprechende Kalihydratmenge zur Verseifnng verwendet worden, also $19.45 \times 0.02577=0.5012 \mathrm{Grm}$. KHO. Die Verseifungszahl ist sonach

$$
501 \cdot 2: 2 \cdot 5715=194 \cdot 9 .
$$

1 Vergl. auch Benedikt: Analyse der Fette und Wachsarten, pag. 116. 2 Li e bi g's Ann. 223, 269. 
Das Moleculargewicht $(\boldsymbol{M})$ des Essigsäureesters ergibt sich aus der Proportion:

$$
M: 56 \cdot 1=1000: 194 \cdot 9,
$$

daraus folgt $M=287 \cdot 7$, während es sich ans der Formel $\mathrm{C}_{16} \mathrm{H}_{33} \mathrm{O} \cdot \mathrm{C}_{2} \mathrm{H}_{3} \mathrm{O} \mathrm{zu} 284$ berechnet.

Von dem in dieser Weise ermittelten Moleculargewicht des Essigsäureesters hat man in allen Fällen 42 (entsprechend $\mathrm{C}_{2} \mathrm{H}_{2} \mathrm{O}$ ) abzuziehen, $\mathrm{um}$ das gesuchte Molecnlargewicht des Alkohols zu finden!

In gleicher Weise wurde das Moleculargewicht des Cholesterins ermittelt. Die Bestimmung ergab $M=368 \cdot 4$, während das aus der Formel $\mathrm{C}_{26} \mathrm{H}_{44} \mathrm{O}$ berechnete Moleculargewicht 372 ist.

\section{Untersuchung von Oxysänren.}

Die Sänrezahl und daraus das Moleculargewicht organischer Säuren lässt sich bekanntlich sehr rasch durch Titration der weingeistigen Lösung von circa 5 Grm. der Säure mit wässriger $1 / 2$-Normallauge unter Anwendung von Phenolphtalëin als Indicator ermitteln. Will man erfahren, wie viele Hydroxylgruppen in der Formel der Säure enthalten sind, so acetylirt man die Säure in gewohnter Weise, reinigt das Product durch wiederholtes Anskochen mit Wasser, dem man zum Schlusse noch ein Umkrystallisiren ans Weingeist folgen lassen kann, und bestimmt nunmehr die Säurezahl und die Verseifungszahl der acetylirten Säure. Je nachdem die Acetylzahl 1, 2, $3 \ldots \ldots$ n-mal so gross als die Säurezahl ist, enthält die Verbindung $1,2,3, \ldots \mathrm{n}$ Acetylgruppen, respective die nicht acetylirte Säure Hydroxylgruppen.

Will man dieses Verfahren zur Untersuchung von aromatischen Säuren, Farbstoffen etc. anwenden, so muss man zurerst die ursprüngliche, nicht acetylirte Substanz auf ihr Verhalten gegen alkoholische Kalilauge prüfen. Dazu verfährt man genau wie bei der Bestimmung der Verseifungszahl. Das Verfahren ist anwendbar, wenn dabei die Verseifungszahl 0 gefunden wird, oder weun sich eine in mehreren Versuchen constante Verseifungszahl ergibt. Wir behalten uns vor, uber eine Reihe derartiger Versuche demnächst ausfuhrlich zu berichten. 
Als Beispiel für die Untersuchung von Oxyfettsäuren sei die Dioxyst earins äure angefiihrt.

\begin{tabular}{|c|c|c|c|c|c|}
\hline \multirow{2}{*}{\multicolumn{4}{|c|}{ Säurezahl der Acetyldioxystearinsäure }} & Gefunden: & Berechnet \\
\hline & & & & $\ldots 139 \cdot 0$ & $140 \cdot 2$ \\
\hline Verseifungszahl & 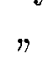 & & $"$ & $\ldots 412 \cdot 2$ & $420 \cdot 6$ \\
\hline Acetylzahl. & & & & $\ldots 273 \cdot 2$ & $280 \cdot 4$ \\
\hline
\end{tabular}

Somit verhält sich die gefundene Acetylzahl zur Säurezahl nahezu wie $2: 1$.

\section{Untersuchung von Fetten.}

Durch Anwendung dieses Verfahrens lässt sich eine Liicke ausfitlen, welche die Analyse der Fette bisher aufwies.

Die natiirlichen Fette bestehen, wenn wir von wachsartigen Beimengungen und geringen Mengen von Kohlenwasserstoffen absehen, aus Glyceriden von flichtigen, wasserlöslichen und nichtflüchtigen, unlöslichen Fettsäıren. Die nichtflüchtigen Fettsäuren kann man in gesättigte und ungesättigte unterscheiden oder die Eintheilung in der Weise vornehmen, dass man die Oxyfettsäuren jenen Säuren gegenuiber stellt, welche keine Hydroxylgruppen enthalten.

Die Fettanalyse begrtindet ihre "quantitativen Reactionen" zur Unterscheidung der Fette auf dem grösseren oder geringeren Gehalt derselben an den genannten Gruppen von Fettsäuren.

Das Reichert'sche Verfahren liefert ein Mass für den Gehalt an flüchtigen Fettsäuren.

Die He hner'sche Methode lehrt die Menge der unlöslichen Fettsäuren bestimmen.

Das nach v. Hübl gemessene Jodad ditionsvermögen gestattet, Schlüisse auf den Gehalt eines Fettes an ungesättigten Säuren zu ziehen.

Nur zur Bestimmung des Gehaltes an Oxyfettsäuren hatte man bisher kein Hilfsmittel. Durch Anwendung unserer Methode lässt sich der Hydroxylgehalt und, wenn die Natur der Oxysäure bekannt ist, auch der Gehalt des Fettes an dieser Säure genau ermitteln. Man ist dann im Stande zu entscheiden, ob ein Fett iiberhaupt Oxysänren enthalte, Fette von bekanntem Oxyfett- 
sïuregehalt zu identificiren und in einigen Fällen Verfälschungen qualitativ und quantitativ nachuweisen.

Wir haben vorläufig die wichtigsten Öle in den Bereich unserer Untersuchung gezogen.

$100 \mathrm{Grm}$. des Öles werden mit $70 \mathrm{Grm}$. in $50 \mathrm{CCm}$. Wasser gelösten Kalihydrates und $150 \mathrm{CCm}$. starken Weingeists bis zur rollständigen Verseifung am Rückflusskühler gekocht. Der Kolbeninhalt wird in eine geräumige Schale ausgegossen, mit einem Liter Wasser verdünnt, mit verdünnterSchwefelsäure angesäuert und so lange gekocht, bis die Fettsäuren als vollkommen klare Schichte oben aufschwimmen und der Alkobol rertrieben ivt.tDie Fettschichte wird noch zweimal mit Wasser ansgekocht, daun durch Abhebern oder mittelst des Scheidetrichters rom Wasser getrennt und in ein kleines Becherglas gegossen, aus welchem sie nach dem völligen Absetzen des Wassers im Lufttrockenkasten bei einer ihren Schmelzpunkt um $20-30^{\circ}$ ïbersteigenden Temperatur auf ein trockenes Filter gegossen werden.

50 Grm. der Fettsäuren werden sodann mit 40 Grm. Essigsäıreanlıd rid zwei Stunden in einem Kölbchen mit Rückflussrohr gekocht, die Mischung in ein hohes Becherglas von einem Liter Inhalt entleert, mit 500-600 CCm. heissem Wasser ibergossen und gekocht ${ }^{1}$. Um ein Stossen der Flüssigkeit zu vermeiden, leitet man durch ein nahe dem Boden des Bechers miundendes Capillarrohr einen langsamen Kohlensäurestrom ein. Nach einiger Zeit hebert man das Wasser ab und kocht noch dreimal mit der gleichen Wassermenge aus. Daun ist, wie man sich durch Prüfung mit Lackmuspapier überzeugen kann, alle Essigsäure entfernt. Endlich filtrirt man die acetylirten Säuren im Luftbate durch ein trockenes Filter und wägt $4-5 \mathrm{Grm}$. zur liestimmung der Säurezahl, 2-3 Grm. zur Bestimmung der Verseifungszahl ab. Bei Ricinusöl wird man zweckmässig die

1 Essigsäureanhydrid wird durch warmes Wasser sehr rasch zerlegt. Versuche, die Entfernung der Essigsäure dadurch zu beschleunigen, dass m:n nach dem Kochen mit Essigsäureanhydrid statt in Wasser in verdünnten Weingeist eingoss, haben ergeben, dass sich dabei ein Theil der Fettsäure leicht ätherificirt. 
doppelte Menge alkoholischer Kalilauge, also $50 \mathrm{CCm}$. zur Verseifung verwenden.

In der folgenden Tabelle sind die bei der Untersuchung einiger Öle gefundenen Zahlen zusammengestellt.

Die erste Columne enthält die Säurezahlen der nicht acetylirten Fettsäuren, aus welchen nach der Formel $\mathrm{M}=56100: \mathrm{S}$ die in der zweiten Columne stehenden mittleren Moleculargewichte berechnet sind. Die Säurezahlen des Rüböles und Ricinusöls sind auffallend niedrig. Es steht dies in voller Übereinstimmung mit den ron Valenta ${ }^{1}$ ermittelten Verseifungszahlen dieser Öle. Der Grund dieser Erscheinung liegt in dem hohen Moleculargewicht der darin enthaltenen Fettsäuren, der Brassicasäure und Ricintısölsäure.

Die dritte, vierte und fünfte Columne enthalten die Säurezahlen, Verseifungs- und Acetylzahlen der acetylirten Fettsäuren. Die Betrachtung der Acetylzahlen lehrt, dass nur das Ricinusöl mit der Zahl 153.4 einen grossen Gehalt an Oxyfettsäuren besitzt, dass aber auch andere öle, insbesondre Cottonöl, Mohnöl und Sesamöl, nicht ganz zu vernachlässigende Mengen solcher Säuren enthalten.

Das Verfahren lässt sich somit sehr gut zur Untersuchung von Ricinusöl anwenden, indem es ermöglicht, die Abwesenheit ron Verfälschungen zu constatiren und die Quantität beigemischter öle genan zu bestimmen, ila schon ein Zusatz von nur fünf Procent eines anderen öles die Acetylzahl um 7.5 Einheiten erniedrigt.

Die Identifirung von Cottonöl kann durch Bestimmung der Acetylzahl leicht bewirkt werden.

Wir sind damit beschäftigt, das Verhalten der festen Fette und Wachsarten bei dieser Reaction zu studiren und deren Anwendbarkeit zur Untersuchung solcherProducte der Fettindustrie zu prüfen, welche Oxyfettsäuren enthalten, so des Türkischrothöles und des Destillat-Stearins.

1 Dingler's Journal 249, 271. 
R. Benedikt u. F. Ulzer, Acetylverbindungen u. s. w.

\begin{tabular}{|c|c|c|c|c|c|}
\hline \multirow{2}{*}{ Fettsäuren ans : } & \multicolumn{2}{|c|}{ Nicht acetylirt } & \multicolumn{3}{|c|}{ Acetylirt } \\
\hline & $\begin{array}{l}\text { Säure- } \\
\text { zahl }\end{array}$ & $\begin{array}{c}\text { Mole- } \\
\text { cular- } \\
\text { gewicht }\end{array}$ & $\begin{array}{l}\text { Säure- } \\
\text { zahl }\end{array}$ & $\begin{array}{l}\text { Versei- } \\
\text { fungs- } \\
\text { zahl }\end{array}$ & $\begin{array}{c}\text { Acetyl- } \\
\text { zahl }\end{array}$ \\
\hline Arachisöl & $198 \cdot 8$ & $282 \cdot 2$ & $193 \cdot 3$ & $196 \cdot 7$ & $3 \cdot 4$ \\
\hline Cottonöl. & $199 \cdot 8$ & $280 \cdot 8$ & $195 \cdot 7$ & $212 \cdot 3$ & $16 \cdot 6$ \\
\hline Crotonöl & $201 \cdot 0$ & $279 \cdot 1$ & $195 \cdot 7$ & $204 \cdot 2$ & 8.5 \\
\hline Hantöl & $199 \cdot 4$ & $281 \cdot 3$ & $196 \cdot 8$ & $204 \cdot 3$ & $7 \cdot 5$ \\
\hline Leinöl & $201 \cdot 3$ & $278 \cdot 7$ & $196 \cdot 6$ & $205 \cdot 1$ & $8 \cdot 5$ \\
\hline Mandelöl & $201 \cdot 6$ & $278 \cdot 3$ & $196 \cdot 5$ & $2012 \cdot 3$ & $5 \cdot 8$ \\
\hline Mohnöl & $200 \cdot 6$ & $279 \cdot 7$ & $194 \cdot 1$ & $207 \cdot 2$ & $13 \cdot 1$ \\
\hline Nussöl & $204 \cdot 8$ & $273 \cdot 9$ & $198 \cdot 0$ & $205 \cdot 6$ & $7 \cdot 6$ \\
\hline Olivenöl & $197 \cdot 1$ & $284 \cdot 6$ & $197 \cdot 3$ & $202 \cdot 0$ & $4 \cdot 7$ \\
\hline Pfïrsichkernöl & $202 \cdot 5$ & $277 \cdot 0$ & $196 \cdot 0$ & $202 \cdot 4$ & $6 \cdot 4$ \\
\hline Ricinusöl & $177 \cdot 4$ & $316 \cdot 2$ & $142 \cdot 8$ & $296 \cdot 2$ & $153 \cdot 4$ \\
\hline Rüböl & $182 \cdot 5$ & $307 \cdot 4$ & $178 \cdot 5$ & $184 \cdot 8$ & $6 \cdot 3$ \\
\hline Sesamöl & $200 \cdot 4$ & $279 \cdot 9$ & $192 \cdot 0$ & $203 \cdot 5$ & $11 \cdot 5$ \\
\hline
\end{tabular}

